UUITP-08/97

10A 97-06

6 May 1997

\title{
Multidimensional Calogero systems from matrix models
}

\author{
Alexios P. Polychronakos ${ }^{\dagger}$ \\ Theoretical Physics Dept., University of Ioannina \\ 45110 Ioannina, Greece \\ and \\ Theoretical Physics Dept., Uppsala University \\ S-751 08 Uppsala, Sweden
}

\begin{abstract}
We show that a particular many-matrix model gives rise, upon hamiltonian reduction, to a multidimensional version of the Calogero-Sutherland model and its spin generalizations. Some simple solutions of these models are demonstrated by solving the corresponding matrix equations. A connection of this model to the dimensional reduction of Yang-Mills theories to $0+1$-dimensions is pointed out. In particular, it is shown that the low-energy dynamics of D0-branes in sectors with nontrivial fermion content is that of spin-Calogero particles.
\end{abstract}

$\dagger$ poly@calypso.teorfys.uu.se 
The quest for integrable (non-relativistic) particle systems in more than one spatial dimension is often frustrating. In general, no nontrivial such systems exist (with the exception of some isolated few-body cases), that is, systems with a nonquadratic potential and which are not a repackaging of one-dimensional degrees of freedom. In one dimension, on the other hand, a whole class of integrable manybody systems is known, namely the Calogero model and its various generalizations (also known as Calogero-Sutherland-Moser systems) [1-3]. The question then is to what extent these models remain solvable, if at all, in higher dimensions.

A particularly fruitful approach to Calogero-like systems is though matrix models, in which the particle positions are regained as the eigenvalues of some appropriate matrix $[4,5]$. The integrability, as well as the solutions of the equations of motion, are simpler to obtain this way. It would seem, then, that this is the most promising route to systems of higher dimension. The purpose of this note is to show that, indeed, appropriate matrix models give rise, under hamiltonian reduction, to multidimensional many-body systems of the Calogero type. These matrix models are not in general integrable, and therefore we do not expect the corresponding particle systems to be integrable either. The hope is, nevertheless, that the (inherently simpler) matrix models dynamics will allow for a better study of the dynamics of the particle systems.

The starting point will be a many-matrix model consisting of $d$ time-dependent hermitian $N \times N$ matrices $M_{i}, i=1, \ldots d$, which we will also represent as a vector of matrices $\vec{M}$. The action will be the usual kinetic term for each matrix plus some potential invariant under simultaneous unitary conjugation of the matrices. The eigenvalues of $M_{i}$ then will be interpreted as the $i$-component of the position vectors of $N$ particles moving in flat $d$-dimensional space. For this interpretation to be possible, however, the matrices must be simultaneously diagonalizable, else there is no invariant association of the $n$-th eigenvalue of the matrices as the coordinates of the same particle. The eigenvalues of all $M_{i}$ can be simultaneously permuted with a common unitary transformation, which corresponds to having identical particles. 
So we write the lagrangian

$$
L=\operatorname{tr}\left\{\sum_{i} \frac{1}{2} \dot{M}_{i}^{2}+i \sum_{i j} \frac{1}{2} \Lambda_{i j}\left[M_{i}, M_{j}\right]\right\}-V(\vec{M})
$$

where overdot stands for time derivative. $L_{i j}=-L_{j i}$ is an antisymmetric set of $d(d-1) / 2$ hermitian matrices serving as Lagrange multipliers for the commutativity constraint between the $M_{i}$. The potential $V(\vec{M})=V\left(U^{-1} \vec{M} U\right)$ can be any real function of the $M_{i}$ invariant under simultaneous unitary transformations of the $M_{i}$. The form $V=\operatorname{tr} V(\vec{M})$, where $V(\vec{x})$ is some real scalar function on $R^{d}$, will be assumed in what follows, which leads to an external potential $V(\vec{x})$ for the particles. The harmonic oscillator potential $V(\vec{x})=\frac{1}{2} \omega^{2} \vec{x}^{2}$, corresponding to the matrix potential $V=\frac{1}{2} \omega^{2} \sum_{i} \operatorname{tr} M_{i}^{2}$, is the simplest example.

For a central potential the model is invariant under $S O(d)$ rotations $R_{i j}$ :

$$
M_{i} \rightarrow R_{i j} M_{j}, \quad \Lambda_{i j} \rightarrow R_{i k} R_{j l} \Lambda_{k l}
$$

As a result, there is a conserved angular momentum

$$
J_{i j}=\operatorname{tr}\left\{M_{i} \dot{M}_{j}-M_{j} \dot{M}_{i}\right\}
$$

Time translation invariance implies the conservation of energy

$$
E=\operatorname{tr}\left\{\sum_{i} \frac{1}{2} \dot{M}_{i}^{2}+V\left(\left\{M_{i}\right\}\right)\right\}
$$

The invariance of (1) under simultaneous conjugation of all matrices by a timeindependent unitary matrix implies the existence of a conserved matrix "angular momentum"

$$
K=i \sum_{i}\left[M_{i}, \dot{M}_{i}\right]
$$

$K$ is a traceless hermitian matrix. Choosing it to have the "minimal" form where 
all its eigenvalues are equal but one, that is,

$$
K_{m n}=\ell\left(\delta_{m n}-u_{m}^{*} u_{n}\right), \sum_{m}\left|u_{m}\right|^{2}=N
$$

where $u$ is a fixed $N$-vector, will lead to Calogero dynamics for the eigenvalues of $M_{i}$, just as in the one-dimensional case,

The equations of motion are

$$
\ddot{M}_{i}+V_{, i}(\vec{M})+i \sum_{j}\left[\Lambda_{i j}, M_{j}\right]=0
$$

plus the constraint

$$
\left[M_{i}, M_{j}\right]=0
$$

The constraint implies that $M_{i}$ can all be diagonalized with a common timedependent unitary rotation $U(t)$ :

$$
M_{i}=U^{-1} X_{i} U, \quad X_{i}=\operatorname{diag}\left(x_{i, 1}, \ldots x_{i, N}\right)
$$

In terms of (9) the equations of motion acquire the form

$$
\ddot{X}_{i}+2\left[\dot{X}_{i}, A\right]+\left[X_{i}, \dot{A}\right]+\left[\left[X_{i}, A\right], A\right]+V_{, i}(\vec{X})+i \sum_{j}\left[\tilde{\Lambda}_{i j}, X_{j}\right]=0
$$

where $\tilde{\Lambda}=U \Lambda U^{-1}$ and $A=\dot{U} U^{-1}$ is the "gauge potential" generated by the time variance of $U$.

We now recall that the commutator of a diagonal matrix with any matrix has zero diagonal elements, since

$$
[D, B]_{m n}=\left(d_{m}-d_{n}\right) B_{i j}, \quad \text { if } D=\operatorname{diag}\left(d_{1}, \ldots d_{N}\right)
$$

Therefore, isolating the diagonal terms in (10), only the first, fourth and fifth term 
contribute and we have

$$
\ddot{x}_{i, m}+\sum_{n} 2\left(x_{i, m}-x_{i, n}\right) A_{m n} A_{n m}+V_{, i}\left(\vec{x}_{m}\right)=0
$$

Plugging the form (6) and (9) in (5), on the other hand we have

$$
i \sum_{i}\left(x_{i, m}-x_{i, n}\right)^{2} A_{m n}=\ell\left(\delta_{m n}-\tilde{u}_{m}^{*} \tilde{u}_{n}\right)
$$

where $\tilde{u}=U u$. For $m \neq n$ and $m=n$ we obtain the relations for $A_{m n}$ and $\tilde{u}_{m}$, respectively

$$
A_{m n}=\frac{i \ell \tilde{u}_{m}^{*} \tilde{u}_{n}}{\left(\vec{x}_{m}-\vec{x}_{n}\right)^{2}}, \quad\left|\tilde{u}_{m}\right|^{2}=1
$$

Plugging these in (12) and calling $\left(x_{1, m}, \ldots x_{d, m}\right)=\vec{x}_{m}$ we finally obtain

$$
\ddot{\vec{x}}_{m}-2 \ell^{2} \sum_{n \neq m} \frac{\vec{x}_{m}-\vec{x}_{n}}{\left(\vec{x}_{m}-\vec{x}_{n}\right)^{4}}+\vec{\nabla} V\left(\vec{x}_{m}\right)=0
$$

which is the equation of motion for the positions of particles $\vec{x}_{m}$ in an external potential $V(\vec{x})$ and interacting through a $d$-dimensional two-body inverse square potential $\ell^{2} / x^{2}$, that is, a $d$-dimensional generalization of the Calogero model. The key elements in the derivation are that the Lagrange multiplier term does not influence the eigenvalue equations of motion and that the angular part reproduces the rotationally invariant $d$-dimensional inverse-square potential. Note that the energy (4) and angular momentum (3) become the corresponding quantities of the particle system in the constraint subspace, that is

$$
\begin{gathered}
E=\sum_{m} \frac{1}{2} \dot{\vec{x}}_{m}^{2}+\sum_{m \neq n} \frac{\ell^{2}}{\left(\vec{x}_{m}-\vec{x}_{n}\right)^{2}}+\sum_{m} V\left(\vec{x}_{m}\right) \\
J_{i j}=x_{i} \dot{x}_{j}-x_{j} \dot{x}_{i}
\end{gathered}
$$

If the "angular momentum" $K$ is not in the "minimal" form (6), it will enter the equations for the eigenvalues in a nontrivial way and will give rise to multidimensional generalizations of the 'spin-Calogero' model [6-9]. To see this, we point 
out that the restriction of the Hamiltonian in the constraint subspace $\left[M_{i}, M_{j}\right]=0$ takes the form

$$
H=\sum_{m} \frac{1}{2} \dot{\vec{x}}_{m}^{2}+\sum_{m \neq n} \frac{\tilde{K}_{m n} \tilde{K}_{n m}}{\left(\vec{x}_{m}-\vec{x}_{n}\right)^{2}}+\sum_{m} V\left(\vec{x}_{m}\right)
$$

where $\tilde{K}=U K U^{-1}$. As usual, $\tilde{K}_{m n}$ Poisson-commute to the $S U(N)$ algebra and can be recast into internal degrees of freedom ("spin") for the particles [10]:

$$
\tilde{K}_{m n}=\sum_{a=1}^{p} S_{m}^{a} S_{n}^{a}
$$

To study the matrix equations of motion we specify to the minimum nontrivial dimensions $d=2$ and to the rotationally invariant harmonic external potential $V(\vec{x})=\frac{1}{2} \omega^{2} \vec{x}^{2}$. Defining the non-hermitian matrix $M=M_{1}+i M_{2}$, the equations of motion and constraint become

$$
\ddot{M}+[\Lambda, M]+\omega^{2} M=0, \quad\left[M, M^{\dagger}\right]=0
$$

while the "angular momentum" K takes the form

$$
K=i\left[M^{\dagger}, \dot{M}\right]
$$

in the constraint subspace. Solving the two-dimensional Calogero model amounts to finding solutions of the above matrix equations for $M$ with the form (6) for $K$.

The simplest possible class of solutions is the one with $\Lambda=0$. It can be shown, however, that these solutions correspond to linear motion of the particles and the system reduces to the one-dimensional Calogero model. The solution to the equations of motion is

$$
M=A e^{i \omega t}+B^{\dagger} e^{-i \omega t}
$$

where the matrices $A, B$, to satisfy the commutativity and "angular momentum" 
constraints, must obey

$$
[A, B]=0, \quad\left[A, A^{\dagger}\right]=\left[B, B^{\dagger}\right]=\frac{K}{2 \omega}
$$

with $K$ as in (6). In terms of the new matrices $Q=A+B^{\dagger}$ and $P=i \omega\left(A-B^{\dagger}\right)$ (representing the position and velocity matrices at $t=0$ ) relations $(23)$ become

$$
\left[Q, Q^{\dagger}\right]=\left[P, P^{\dagger}\right]=0, \quad\left[Q, P^{\dagger}\right]=-i K
$$

This tells us that $P$ and $Q$, although non-hermitian, can each be diagonalized with a unitary rotation, with complex eigenvalues (representing the initial positions and velocities of the particles on the complex plane). Choosing a basis where $Q$ is diagonal with eigenvalues $q_{m}$, we deduce from the last relation in (24) that the matrix elements of $P$ are

$$
P_{m n}=i p_{m} \delta_{m n}+\frac{i \ell}{q_{m}^{*}-q_{n}^{*}}\left(1-\delta_{m n}\right)
$$

where we used $\left|u_{m}\right|=1$ in the $Q$-diagonal basis and further chose the phases of the states such that $u_{m}=1$. From $\left[P, P^{\dagger}\right]=0$ now we obtain

$$
\frac{p_{m}-p_{n}}{q_{m}-q_{n}}=\text { real }, \quad \sum_{k \neq m, n} \frac{1}{\left(q_{m}-q_{k}\right)\left(q_{m}^{*}-q_{n}^{*}\right)}=\text { real }
$$

By using the invariance of the equations by a shift of $Q$ and $P$ by a multiple of the unit matrix (which is related to the fact that the center-of-mass motion decouples from the relative motion) we can always choose $q_{1}=p_{1}=0$. Then the first relation above implies that all $q_{m}$ are collinear (i.e., $q_{m} / q_{n}=$ real) unless $p_{m}=a q_{m}$ for some real $a$. The second relation, however, is satisfied only if the $q_{m}$ are collinear. By the first relation, $p_{m}$ will also be collinear with $q_{m}$. Therefore, we see that the one-dimensional Calogero model is included in the $\Lambda=0$ sector of the general model. 
The other case in which the equations of motion have an obvious solution is when $\Lambda=$ constant. Choosing a basis in which $\Lambda$ is diagonal, with (real) eigenvalues $\lambda_{n}$, we have

$$
\ddot{M}_{m n}+\omega_{m n}^{2} M_{m n}=0, \quad \omega_{m n}=\omega^{2}+\lambda_{m}-\lambda_{n}
$$

which has as solutions (we assume $\omega_{m n}^{2}>0$ )

$$
M_{m n}=A_{m n} e^{i \omega_{m n} t}+B_{m n}^{\dagger} e^{-i \omega_{m n} t}
$$

The task of finding the most general $A_{m n}, B_{m n}$ which satisfy the commutativity and "angular momentum" constraints is not trivial. We demonstrate here a particularly simple solution, namely

$$
\begin{aligned}
& A_{m n}=A_{m} \delta_{m n}+a_{n} \delta_{m, n+1}, \quad B_{m n}=B_{n} \delta_{m n} \\
& \left|a_{n}\right|=a, \quad \omega_{n+1, n+2}-\omega_{n, n+1}=\frac{\ell}{a^{2}}
\end{aligned}
$$

The last constraint for $\omega_{n, n+1}$ translates into $N-1$ algebraic equations for the $N-1$ variables $\lambda_{n}-\lambda_{n+1}$ (clearly $\Lambda$ can be shifted by any multiple of the unit matrix). It is obvious that the diagonal part $A_{m}, B_{m}$ represents a general motion of the decoupling center of mass. The eigenvalues of the off-diagonal part of $A$ are the $N$-th roots of $a_{1} a_{2} \cdots a_{N}$. So the off-diagonal part of $M$ has eigenvalues

$$
z_{m}=x_{m}+i y_{m}=a e^{i\left(\frac{2 \pi m}{N}+\omega_{r} t\right)}, \quad \omega_{r}=\sum_{n} \omega_{n, n+1}=\omega_{12}+(N-1) \ell / a^{2}
$$

Therefore the relative motion is one in which the particles are regularly positioned on a circle of radius $|a|$ and rotate with constant angular velocity $\omega_{r}$.

The above model can be generalized to one with unitary matrices. Omitting the details of the calculation, we simply state the result. The lagrangian of the 
model is

$$
L=\operatorname{tr}\left\{\sum_{i} \frac{1}{2} R_{i}^{2} \dot{U}_{i}^{\dagger} \dot{U}_{i}+i \sum_{i j}\left(\Lambda_{i j}\left[U_{i}, U_{j}\right]+\Lambda_{i j}^{\dagger}\left[U_{i}^{\dagger}, U_{j}^{\dagger}\right]\right)\right\}-V\left(\left\{U_{i}\right\}\right)
$$

where again $V$ is some real conjugation-invariant potential. The Lagrange multiplier matrices $\Lambda_{i j}=-\Lambda_{j i}$ are not hermitian, but the constraints arising from the variation of $\Lambda_{i j}$ and $\Lambda_{i j}^{\dagger}$ are compatible (in fact, equivalent) for unitary $U_{i}$. The eigenvalues of $U_{i}$, written as $\exp \left(i x_{i, m} / R_{i}\right)$ represent coordinates of particles on a $d$-dimensional torus of radii $R_{i}$. Upon choosing the "angular momentum"

$$
i \sum_{i} R_{i}^{2}\left[U_{i}^{\dagger}, U_{i}\right]=\ell\left(1-u u^{\dagger}\right)
$$

as before, the $\vec{x}_{n}$ move like particles on the torus in an external potential $V\left(\vec{x}_{n}\right)$ and interacting through a periodic generalization of the $d$-dimensional two-body inverse-square potential

$$
V\left(\vec{x}_{n}-\vec{x}_{m}\right)=\frac{\ell}{\sum_{i} \pi^{2} R_{i}^{2} \sin ^{2} \frac{x_{i, m}-x_{i, n}}{\pi R_{i}}}
$$

Similarly, the $d$-dimensional generalization of the inverse-sinh model can be obtained by taking $R_{i} \rightarrow i R_{i}$.

It is interesting to note a connection of the models presented here with the matrix model obtained as the dimensional reduction of $(\mathrm{d}+1)$-dimensional YangMills theory. In the $A_{o}=0$ gauge, this model is essentially the kinetic part of the above model plus a potential of the form

$$
\operatorname{tr} \sum_{i j} \frac{1}{2}\left[M_{i}, M_{j}\right]^{2}
$$

One can also consider the supersymmetric version of this model, where there are also appropriate fermionic terms $[11,12]$. In fact, this model has also appeared as 
a regularization of the light-cone membrane action[13]. For $d=9$ it describes the low-energy dynamics of D-particles [14-16] and has recently been proposed as a matrix model description of M-theory in the large- $N$ limit [17]. This model can be thought of as our model where a mass therm has been given to the Lagrange multiplier matrices $\frac{1}{2} \epsilon^{2} \Lambda_{i j}^{2}$. Integrating out $\Lambda_{i j}$ (that is, solving their equation of motion) generates the above potential term (34) with a coefficient $1 / \epsilon^{2}$. For $\epsilon \rightarrow 0$, corresponding to our model, the strength of the potential grows very large. Therefore, our model can be though of as the low-energy limit of the M-theory model, when the $M_{i}$ are at the minimum of the potential (34), that is, they commute. In the super-Yang-Mills (M-theory) model there is also the Gauss law constraint

$$
\sum_{i} i\left[M_{i}, \dot{M}_{i}\right]+\sum_{i} \Theta_{i} \Theta_{i}^{T}=0
$$

where $\Theta_{i}$ are Majorana-Weyl spinors, the superpartners of $M_{i}$. The bosonic part of (35) is just the "angular momentum" $K$. The fermionic part of (35) provides real representations of $S U(N)$, consisting of (the irreducible components of) totally antisymmetric tensor products of adjoint representations ( $\Theta$ transforms in the adjoint of $S U(N)$ ). Consequently, (35) constrains the "angular momentum" $K$ to be in one of these representations, depending on the fermionic sector of the model. Therefore we conclude that the low-energy motion of the eigenvalues of $M_{i}$ (that is, the coordinates of the so-called D0-branes in M-theory) is the one of particles in the $d$-dimensional spin-Calogero model ( $d=9$ for M-theory), where the spin degrees of freedom of the particles, which participate in the dynamics, are determined by the fermionic state of the model through (35) and (19). The physical implications of this fact are left for future study.

We conclude with some remarks. Clearly, there are a lot of unanswered questions here. Firstly, the physical meaning of the Lagrange multiplier matrices $\Lambda_{i j}$ and their role at classifying the types of solutions are yet to be understood. The matrix equations of motion have barely been touched in the general case, and their solutions are unknown. Even in the case $\Lambda=$ constant the general solution has not 
been fully studied. Generalizations of these models involving the Weierstrass function potential could be sought, where instead of a matrix model one would have to deal with an appropriate topological model [18]. In fact, it would be interesting to consider what type of model would give rise to Calogero-type dynamics on a manifold of more general geometry and/or topology. Finally, the quantization of the model, being a constrained system, is a subject of investigation.

\section{REFERENCES}

1. F. Calogero, J. Math. Phys. 10 (1969) 2191 and 2197; 12 (1971) 419; Lett. Nuovo Cimento 13 (1975) 411; F. Calogero and C. Marchioro, Lett. Nuovo Cimento 13 (1975) 383.

2. B. Sutherland, Phys. Rev. A4 (1971) 2019; 5 (1972) 1372; Phys. Rev. Lett. 34 (1975) 1083.

3. J. Moser, Adv. Math. 16 (1975) 1.

4. D. Kazhdan, B. Kostant and S. Sternberg, Comm. Pure Appl. Math. 31 (1978) 481.

5. M.A. Olshanetskii and A.M. Perelomov, Phys. Rep. 71 (1981) 314; 94 (1983) 6.

6. J. Gibbons and T. Hermsen, Physica D11 (1984) 337.

7. S. Wojciechowski, Phys. Lett. A111 (1985) 101.

8. Z.N.C. Ha and F.D.M. Haldane, Phys. Rev. B46 (1992) 9359.

9. J. Minahan and A.P. Polychronakos, Phys. Lett. B302 (1993) 265.

10. J. Minahan and A.P. Polychronakos, Phys. Lett. B326 (1994) 288.

11. M. Claudson and M.B. Halpern, Nucl. Phys. B250 (1985) 689.

12. M. Baake, M. Reinicke and V. Rittenberg, J. Math. Phys. 26 (1985) 1070.

13. B. de Witt, J. Hoppe and H. Nicolai, Nucl. Phys. B305 (1988) 545. 
14. U.H. Danielsson, G. Ferretti and B. Sundborg, Int. Jour. Mod. Phys. A11 (1996) 5463.

15. D. Kabat and P. Pouliot, Phys. Rev. Lett. 77 (1996) 1004.

16. M.R. Douglas, D. Kabat, P. Pouliot and S.H. Shenker, hep-th/9608024.

17. T. Banks, W. Fischler, S.H. Shenker and L. Susskind, hep-th/9610043.

18. A. Gorskii and N. Nekrasov, Theor. Math. Pnys. 100 (1994) 874. 